SCCIDoc

\section{A Conservative Surgical Approach in the Management of Radicular Cysts Associated with Primary Molar Teeth: A Report From 2 Cases}

\section{International Journal of Dentistry and Oral Science (IJDOS) ISSN: 2377-8075}

Senthil Eagappan $\mathrm{AR}^{1 *}$, Sujatha $\mathrm{S}^{2}$, Daya Srinivasan ${ }^{3}$, Divya Natarajan ${ }^{4}$

${ }^{1}$ Professor, Department of Pedodontics \& Preventive Dentistry, Chettinad Dental College \& Research Institute, Kelambakkam, Tamil Nadu, 603103, India.

${ }^{2}$ Consultant-Pediatric Dentist, Elite Dentistry, 48A, PH Road, Kumananchavadi, Chennai, Tamil Nadu, 600056, India.

${ }^{3}$ Professor, Department of Pedodontics \& Preventive Dentistry, Chettinad Dental College \& Research Institute, Kelambakkam, Tamil Nadu- 603103, India.

${ }^{4}$ Lecturer, Department of Pedodontics \& Preventive Dentistry, Chettinad Dental College \& Research Institute, Kelambakkam, Tamil Nadu - 603103, India.

We would like to report on two cases of radicular cysts managed by conservative surgery in children with dental caries infection, presenting with jaw swellings. The Case 1, was a 7 year old boy who had presented with an asymptomatic swelling on the left side of face for a month. The swelling was diffuse, non tender, bony hard and localized to left side body of mandible. Intraoral examination revealed dental caries in lower left molar teeth (numbered as 74,75 ) with obliteration of buccal sulcus indicating buccal cortical plate expansion. (Figures $1 \mathrm{~A} \& 2 \mathrm{~A}$ ). The case 2 was an 8 year old girl, who had presented with a discreet expansion of the body of the mandible on left side resulting in a mild facial asymmetry. As reported, non-painful swelling was first noticed three months earlier, which then progressed slowly to attain the current size. On examination the swelling was hard on palpation in the vicinity of lower left molar tooth (numbered as 75). (ee Figure $1 \mathrm{~B} \& 2 \mathrm{~B})$ In both the cases, radiographic findings revealed a welldefined unilocular radiolucency measuring approximately $25 \mathrm{~mm}$ in its largest diameter, extending from the periapical area of 75 tooth to the mesial aspect of 36 tooth. In the Case 1, expansion of the lesion had displaced the permanent second premolar very close to the lower border of the mandible. (Figures 3A \& 3B) A fine needle aspiration of the lesion fluid revealed a straw colored fluid in both the cases. A provisional diagnosis of radicular cyst was made, based on these findings. Marsupialization was planned keeping in mind the size of the lesion, involvement of permanent tooth bud and proximity to inferior alveolar canal. In both cases, Extraction of the offending tooth was performed under local anesthesia. Then the cyst was exposed surgically and the cystic lining was sutured to alveolar mucosa. The cavity was rinsed with sterile saline and povidone iodine combination and then packed with gauze impregnated with iodoform glycerin paste. A part of cyst lining was obtained for histopathological examination which confirmed the clinical diagnosis. Patients were reviewed and gauze packing changed every two weeks postoperatively for three months, followed by monthly review. In both patients, progressive improvement was observed in facial asymmetry. In the Case 1 , at first year recall clinical and radiological examination revealed good healing of soft tissues, new bone formation in the cystic space, gradual up righting of 44, 45 into their appropriate position and continued root development in 36. (Figures 4A \& 5A) In the Case 2, 35 erupted but in a more buccal position, approximately within 1 year. Orthopantomogram revealed new bone formation and continued root development in 36. (Figures 4B \& 5B)

The Dental (Radicular cysts) are by the most common inflammatory cysts that occur in jaw due to a untreated dental infection or caries tooth $[1,2]$. The frequency of radicular cysts in primary dentition is approximately $0.5-3.3 \%$ [3]. The untreated cyst formation in children may cause abnormal bony expansion and resorption, delayed eruption, malposition of erupting teeth, enamel defects or damage to the developing permanent successors. This justifies need for timely surgical treatment of children with radicular cysts [4]. The clinical presentation of these patients include, pain (infected tooth), swelling, fever, cervical lymphadenopathy and odynophagia (large cyst in molar teeth). This presentation often leads a child to a pediatrician/surgeon than a dental specialist. A diagnosis needs a dental panoramic radiographs, as used in both cases. Treatment options for radicular cysts include extraction of associated teeth and total enucleation in the case of small lesions, marsupialization for decompression of larger cysts, or a combination of the two techniques.

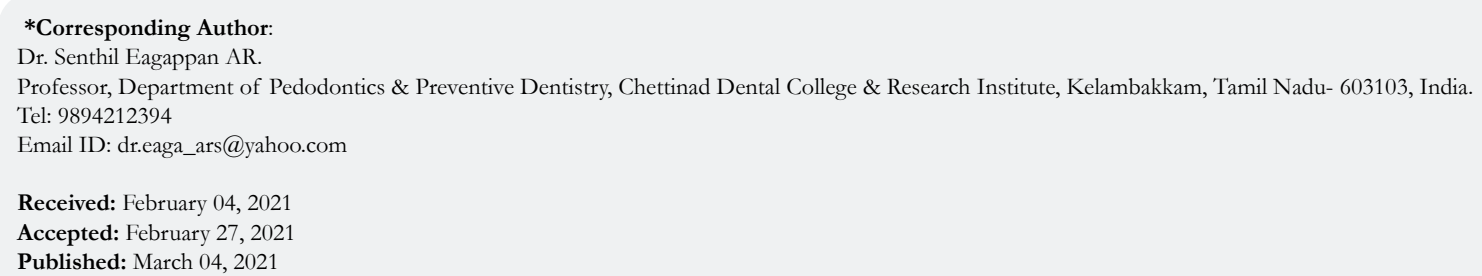

Copyright: Senthil Eagappan $\mathbf{A R}^{\circ}$ 2021. This is an open-access article distributed under the terms of the Creative Commons Attribution License, which permits unrestricted use, distribution and reproduction in any medium, provided the original author and source are credited. 
Figure 1A \& 1B. Preoperative - Extraoral view of the two patients.

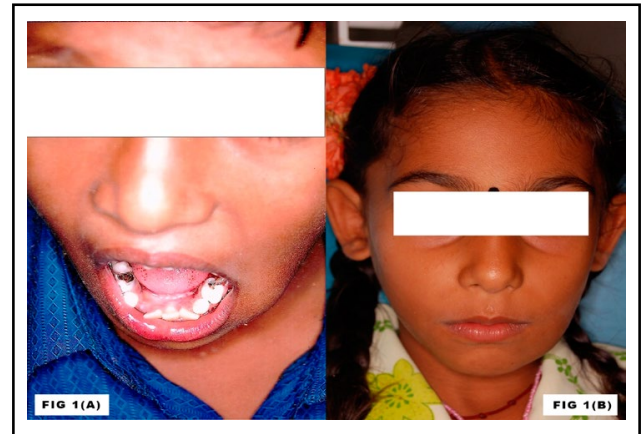

Figure 2 A \& 2B. Preoperative clinical features of the two patients with a Radicular cyst.

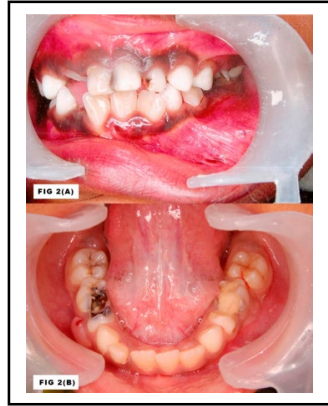

Figure 3A \& 3B. Radiograph before Marsupialization of the radicular cyst exhibiting a well-defined unilocular radiolucency in the periapical area of 75 .

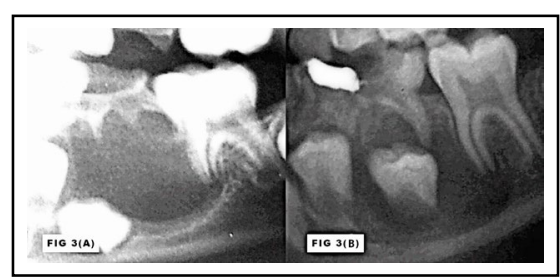

Figure 4A \& 4B. Post-treatment clinical view (fixed space maintainer device in case 1).

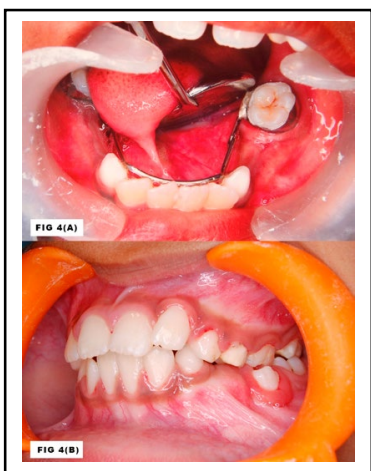

Figure 5A \& 5B. Post-treatment panoramic radiograph..

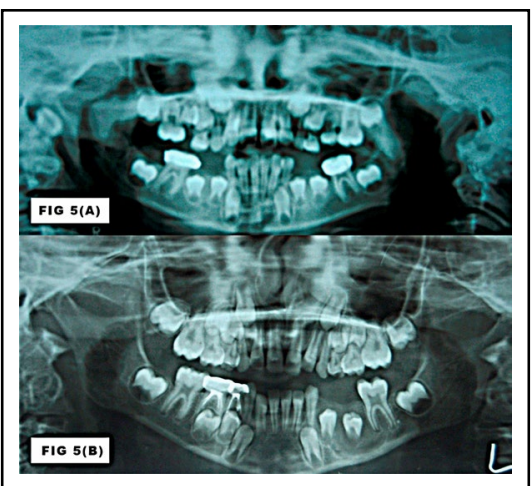


The surgical management of cystic lesions of the jaw is either enucleation or marsupialization. Enucleation is defined as a complete removal of the cystic lining with healing by primary intention. Marsupialization is synonymous with Partsch's operation and is the conversion of a cyst into a pouch [3, 4]. The procedure involves deroofing the outer wall of a cyst and establishing a permanent opening by suturing the remaining cystic wall to the mucosal surface. This in turn would relieve the internal pressure in the cyst and thereby promote healing. The treatment of choice for jaw cysts depends on the size and location of the lesion and its proximity to neighboring vital structures. For the cases reported here, enucleation of the cyst may cause damage to the underlying developing tooth germs (Premolars) or lead to a pathological fracture of mandible. Marsupialization was successful, because of the inherent tendency for the cyst lining to contract, probably due to the myofibroblasts in their walls, once the liquid contents are released [5]. Once the cystic pressure decreases, bony ingrowth with normal bony contour occurs. The macrophages and lymphocytes from inflammation (caused by contamination with oral fluids) release growth factors that enhance bone formation [6].

In both cases presented, new bone formation in the cystic space was visible at the sixth month recall. The disadvantages of marsupulisation includes preservation of pathological tissue inside the jaws without histological examination need for patient's cooperation in cleaning the pathological cavity and follow-up visits [7]. Despite the drawbacks, marsupialization revealed to be more advantageous when considering unnecessary loss of vital teeth and bone obtained with enucleation. Therefore marsupulisation is considered a worthwhile procedure for large infected cyst involving the tooth buds. The post-surgical sites must be evaluated by radiographs for safety of permanent tooth buds and later followed up until eruption of permanent tooth. A 'space maintainer' (as used in case 1) may be needed in some cases to save the space needed for erupting tooth based on age of patient. Thus, the radicular cysts occur after a dental infection in deciduous teeth may be diagnosed by panoramic radiographs and surgically managed by conservative approaches as described. The timing of surgery and follow up till eruption of permanent teeth are goals of compete treatment plan.

\section{References}

[1]. Jones AV, Craig GT, Franklin CD. Range and demographics of odontogenic cysts diagnosed in a UK population over a 30-year period. J Oral Pathol Med. 2006 Sep;35(8):500-7. Pubmed PMID: 16918602.

[2]. Penumatsa NV, Nallanchakrava S, Muppa R, Dandempally A, Panthula P. Conservative approach in the management of radicular cyst in a child: case report. Case Rep Dent. 2013;2013:123148. Pubmed PMID: 23476812.

[3]. RamakrishnaY, VermaD. Radicular cyst associated with a deciduous molar: a case report with unusual clinical presentation. J Indian Soc Pedod Prev Dent .2006;24(3): 158-60. Pubmed PMID: 17065786.

[4]. Mass E, Kaplan I, Hirshberg A. A clinical and histopathological study of radicular cysts associated with primary molars. J Oral Pathol Med. 1995;24(10):458-61. Pubmed PMID: 8600282.

[5]. Neaverth EJ, Burg HA. Decompression of large periapical cystic lesions. J Endodontics. 1982;8(4):175-182. Pubmed PMID: 6951918.

[6]. Sakkas N, Shoeen R, Schulze D, Otten JE, Schmelzeisen R. Obturator after marsupialization of a recurrence of a radicular cyst of the mandible. Oral Surg Oral Med Oral Pathol Oral Radiol Endod. 2007;103(1): e16-18. Pubmed PMID: 17178480.

[7]. Soliman MM, Hassan HD, Elgazaerly H, Sweedan TO. Marsupialization as a treatment modality of large jaw cysts. World Appl Sci J. 2013;21:1752-9.

[8]. Gervasio AM, Silva DA, Taketomi EA, Souza CJ, Sung SS, Loyola AM. Levels of GM-CSF, IL-3, and IL-6 in fluid and tissue from human radicular cysts. J Dent Res.81(1):64-8. Pubmed PMID: 11824415. 